Gastroenterologia. 1948;73:I-IV

\title{
Contents, Vol. 73, 1948
}

\section{INDEX}

Arnous, J, vide Racket, J

Busson, A., vide Racket, J. .

Cramer, R., Talkgranulomatose des Perito < neums , . .129

Finkelstein, Ch., Zur Frage der Behandlung des inoperable $3 / 8 n$

Magenkrebses mit Autovaceinationen von Magensaft des

Kranken ..... . .45

Goldschmíed, A., Contributk > in à I'étude de laglycémie a $\alpha$ eours

de la maladie ulcéreuse de 1 'estomac et du duodenum et des

affections $1 / 2$ flammatoires $\quad 209$

Haak, A., Etude du traitement des uleères gastro-duodénaux 343

Lambling, A. et Soullard, J., Ouelques causes d'échecs des traitements médicaux et chirurgieaux dans l-es uleères gastro-duodénaux 327

L $\psi$ on, E., Adrenalin Discharges in the Pathogenesis of Cardiovascular and Gastroduodenal

Disease ..... 219

Mamie, M., Traitement de 1'ulcère gastro-duodén < al par le Ro-

buden ....157

Plaschkes, S. J., Vagusresektion als Ulcusoperation . ., 240

Policzer, N., Zweizeitige Milzblutungen 30

Racket, J., Busson, A. et Arnous, J., La Polypose colique dis-

séminée 362

Reimann, F., Das „anämische Fieber”. II. Der Ursprung und

die Ursache der abnormen Temperaturen bei der perniziösen

Anämie $56,173,248$

Saegesser, M., Einiges über den Pruritus ani .... 1

Salvesen, H.A., Symptomatic Sprue. Due to Retieulosarco-matosis of the Small Intestine and the Mesentery Gîands . 166

Schnetz, H., Untersuohungen über den Einfluß von Laciofiavin

auf den remalen Diabetes 17

Soullard, J., vide Lambling, A.

Walther, O., Beitrag zur Differentialdiagmose des praepyiori-

schen Uieus und Carcinom 352

Paschoud, H., Premier Congrès de ГAssociation Européenne des Sociétés Nationales de Gastro-Entérolagie. Seances des 23-25 juillet 1948 à Lausanne. Souhaits de bienvenue et introduction 321

Gesellschaftsberichte - Society Transactions - Sociétés

Eighth Meeting of the British Society of Gastro-Enterologists.

London, 7 th and 8 th November 1947 
Editorials

Sérothérapie anti-ulcéreuse

Dysphagia and the Short Oesophagus .

Recherdhes sur l'ulcère aux USA.

Traifcements nouveaux de la tuberculose intestinale

9C $18 i ́$

27:

37:

Referate $\cdot$ Abstracts - Analyses

A. Die gastroenterologischen Zeitschriften - Revues specialises 91, 18

B. Literaturübersicht - Revue de la littérature . . 97, 189, 299, 37

/. Al $\downarrow$ gemeínes - Généralités .... //. Mutidhöhle - Cavitê buccale

Sueise/öhre - O,esophage ....

Magen - Esío,mac

Paẅologische Physiologic - Physio-patholog

Diagnose - Diagnostic ....

Ulcus ventriculi et duodeni .

Carcinoma ......

Gastritis

Gutartige Tumoren - Tumeurs bénignies

Varia

V. Leber - Foie

Diagnose - Diagnostic ....

Hepatitis - Hepatite ....

Cirrhose

Leber und Ernährung - Foie tet alimentation

Varia

VI. Gaílenwege - Voies biliaíres

Ki'a \kheitsbild - Sémiologie .

Therapie - Thérapeutique

Sphincter Oddi - Sphincter d'Oddi

VII. Pancreas

VIII. Diinndariv. - Intestin grêl·e IX. Dickdarm - Colon .....

$\Lambda \backslash$ Peritoneum - Péritoine ....

315.

Buchbesprechungen - Books Review - Livres Nouveaux 320,

Varia ..... 